\title{
Unstable Angina and Renal Colic in a Patient with Ankylosing Spondylitis - A Challenge during the COVID- 19 Pandemic
}

\author{
Azhar Sayyed $^{1}$, Jibril Khalil ${ }^{2}$, Rahul Guhabiswas ${ }^{2}$, and Lalit Kapoor ${ }^{1}$ \\ ${ }^{1}$ Rabindranath Tagore International Institute of Cardiac Sciences \\ ${ }^{2}$ Affiliation not available
}

January 20, 2021

\begin{abstract}
Ankylosing spondylitis (AS) a seronegative inflammatory disease that often presents with co-existing issues like bilateral hip replacements, nephrolithiasis, skin lesions, peripheral vascular disease and coronary artery disease.. We describe a patient with ankylosing spondylitis with bilateral hip replacements in the past who was waiting for an elective coronary artery bypass grafting (CABG) but needed urgent admission with renal colic and unstable angina. In the current COVID pandemic in order to reduce hospital stay and risk of hospital acquired COVID infection we decided to perform simultaneous CABG and removal of ureteric stone. In this case report we discuss the issues in relation to management of patients with coronary artery disease and ankylosing spondylitis.
\end{abstract}

\section{Introduction:}

Ankylosing spondylitis (AS) is a seronegative inflammatory disease that progressively affects the spine and sacroiliac joints. These patients often present with co-existing issues like bilateral hip replacements, nephrolithiasis, skin lesions, peripheral vascular disease and coronary artery disease. (1-5) It is, however, rare to find all of these in one single patient. We describe a patient with ankylosing spondylitis with bilateral hip replacements in the past who was waiting for an elective coronary artery bypass grafting (CABG) but needed urgent admission with renal colic and unstable angina. Synchronous CABG and ureteric stone retrieval along with DJ stent was successfully carried out in this patient with ankylosing spondylitis.

\section{CASE REPORT:}

A 52 year old gentleman presented with renal colic and unstable angina, for which he was admitted at another centre. . His angiogram, there, revealed $70 \%$ distal left main stem disease along with $90 \%$ long segment disease involving proximal LAD, Cx and RCA. He also had impaired LVEF(40\%). He also had significant skin changes in both lower legs, with thread-like veins, and had Doppler confirmed bilateral lower limb peripheral vascular disease and restrictive lung function tests. On ultrasound multiple renal calculi and an obstructive left ureteric calculus, dilatation of the pelvi-calyceal system and mild perinephric collection was observed. Previously he also had bilateral hip replacements done because of inflammatory arthritis.

In the current COVID-19 pandemic admitting patients for surgical procedures is fraught with multiple issues. Pre-hospital quarantine and screening upon admission are some of the measures that are undertaken in elective cases; however, urgent cases like this pose a bigger challenge. In the presence of dual pathology both requiring operative intervention the clinical decision to prioritise one procedure over the other and the sequence of the operationare. important. multiple procedures or prolonged hospital admission makes the patient susceptible to hospital acquired COVID infections. As a result after a multidisciplinary team meeting a decision to perform a synchronous CABG as well as ureteroscopy and double-J( DJ) stenting was 
taken under a single anaesthesia. Simultaneous CABG and ureteric stone retrieval along with DJ stent was successfully carried out in this patient with ankylosing spondylitis.

All standard precautions recommended during aerosol generating procedures were taken during intubation. Because of extreme neck flexion, a decision to perform intubation aided by fibre-optic bronchoscopy was taken at the outset. [Figure 1] A non-kinkable size (7.5Fr) endotracheal tube on a bougie was used. Adequate neck and cervical spine support was provided.CABG was performed first. Midline sternotomy incision was made 2 fingers breadth below the sternal notch to the xiphisternum. The sternum was split using the sternal saw in a reverse fashion to our usual practice, starting at the xiphisternum towards the sternal notch. This was done because of a significantly reduced sterno-mental distance. [Figure 1b] Bilateral internal thoracic arteries were harvested and a total arterial revascularization was performed as an off-pump beating heart procedure. Harvesting the ITA required use of a long tip diathermy as access of the proximal ITA was

As the patient after induction was stable, cysto-ureteroscopy was performed before CABG. The ureteric stone was retrieved and a doubleJ (DJ ) stent was implanted to relieve ureteral obstruction.

The patient made an uneventful recovery and was discharged on day seven.

\section{Discussion}

There are several issues in patients with ankylosing spondylitis undergoing CABG. Intubation in these patients has been historically difficult and fiber optic intubation is often required. In addition, in the current COVID pandemic risk of aerosol generation is excessive during fibre optic bronchoscopy and a prolonged intubation process. This is especially relevant in patients who present urgently without preadmission quarantine or the option of a double screening RT-PCR. As a result adequate protective measures have to be in place to safeguard health care personnel during the intubation process. In order to minimize the risk of aerosol generation we decided to perform the intubation under endoscopic guidance at the very outset. The most experienced anaesthetic consultant available conversant with fibre-optic bronchoscopy performed the procedure. Several pitfalls are identified with airway management and awake fibreoptic intubation is thought to be the safest option that allows continuous neurological monitoring while achieving a definitive airway.(6)

Also, legs should be routinely inspected to identify the suitability of vein harvesting. Skin changes in AS are rare and often limited to Psoriasis but leukocytoclastic vasculitis in AS has also been reported(1) Lipodystrophy as seen in our case renders the vein a poor conduit choice. In this patient, BITA were used partly because of skin changes in both lower limbs, with poor veins that felt narrow and hard from the outside. The patient also had significant peripheral vascular disease. Also, because of the fixed flexion deformity of the cervical spine the access to the proximal aorta is quite limited and hence using BITA precludes the need to access the aorta for construction of proximal anastomosis.

Nephrolithiasis as seen in our patient is a lesser recognized but extremely important association of ankylosing spondylitis and has been considered to be a novel extra-articular manifestation. As seen in our case male patients with AS are at increased risk of nephrolithiasis as seen in our case.(3) It has been reported that compared to the general population patients with AS have almost three-fold increased risk of surgical intervention for kidney stones.(7)

Manubrio-sternal joints are involved in $80 \%$ of patients with AS with features of erosions or fusion and it has been shown that in the eroded joints in ankylosing spondylitis cartilage is replaced by collagenous and fibrous tissue spreading into the bone. (8) The bones can thus be hard to saw through but brittle and may pose significant problems with sternal union. AS also commonly involves the hip joint and usually bilateral hip replacements are required. (5) This poses significant issues with post-operative mobilisation after CABG especially if veins have been harvested from the legs. In addition peripheral vascular disease and associated lipodystrophy of the vein as observed in our case might preclude their usage. (2)

There is also an increased association between AS and coronary artery disease. Based on a meta-analysis conducted it has been seen that a statistically significant increased risk of CAD exists in patients with AS. 
The risk is quantified as $41 \%$ greater than the general population.(1) Spondyloarthritis also predisposes these patients to develop CAD almost 6 years earlier than in the general population. It has been reported to be a stronger predictor of early CABG than most of the traditional cardiovascular risk factors.(9)

The pathophysiology behind the association between AS and CAD is not well-described though an increasing number of evidence are pointing toward the detrimental effect of chronic inflammation to the endothelial cell integrity. It has been demonstrated that endothelial dysfunction and direct endovascular injury from inflammatory cytokine, activated inflammatory cells and oxidative stress can accelerate the progression of atherosclerosis.(1)

As far as the prognosis of CABG is concerned while AS increases the risk of adverse outcomes it is not significantly associated with overall mortality and adverse cardiac outcomes.(10) As a result the patients should not be denied surgical revascularization. Awareness of issues around intubation, choice of conduits, access to the aorta, sternal closure, and mobilisation are important for a satisfactory outcome. Utilization of BITA grafting where possible can ameliorate some of these issues and improve outcomes and minimize the need for reoperation in a challenging subset of patients. .

Figure Legend 


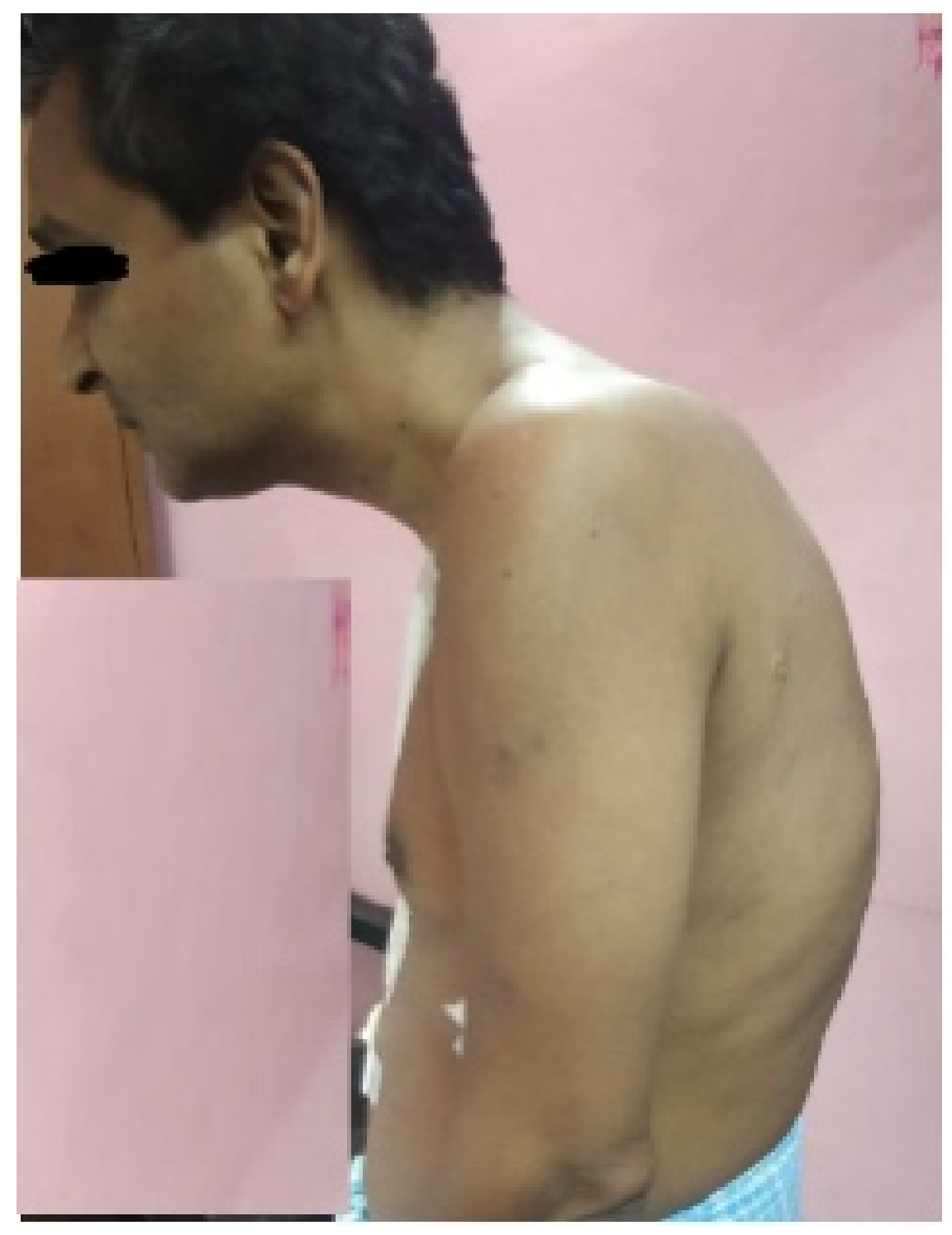




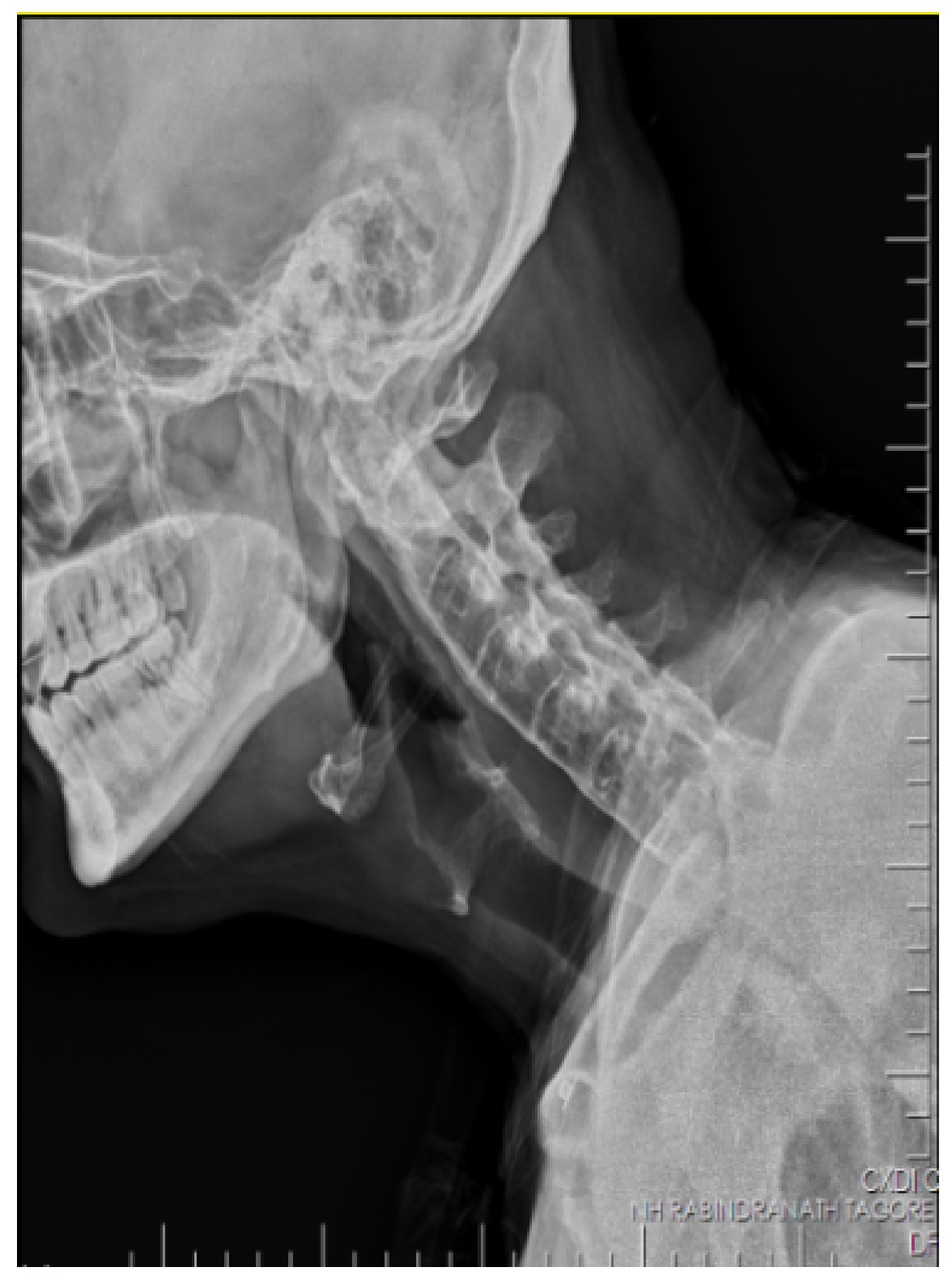

Figure 1 A,B : Fixed flexion deformity of the cervical spine

\section{References:}

1. Ungprasert P, Srivali N, Kitana Mongkolchai W. Risk of coronary artery disease in patients with ankylosing spondylitis: a systematic review and meta-analysis. Ann Transl Med. 2015 Mar;3(4):51.

2. Han C, Robinson DW, Hackett MV, Paramore LC, Fraeman KH, Bala MV. Cardiovascular disease and risk factors in patients with rheumatoid arthritis, psoriatic arthritis, and ankylosing spondylitis. J Rheumatol. 2006 Nov;33(11):2167-72.

3. Jakobsen AK, Jacobsson LTH, Patschan O, Askling J, Kristensen LE. Is Nephrolithiasis an Unrecognized Extra-Articular Manifestation in Ankylosing Spondylitis? A Prospective Population-Based Swedish National Cohort Study with Matched General Population Comparator Subjects. PLoS ONE [Internet]. 2014 [cited 2020 Sep 16];9(11). Available from: https://www.ncbi.nlm.nih.gov/pmc/articles/PMC4244108/ 
4. Ye C, Li W. Cutaneous vasculitis in a patient with ankylosing spondylitis. Medicine (Baltimore) [Internet]. 2019 Jan 18 [cited 2020 Sep 17];98(3). Available from: https://www.ncbi.nlm.nih.gov/pmc/articles/PMC6370148/

5. Lin D, Charalambous A, Hanna SA. Bilateral total hip arthroplasty in ankylosing spondylitis: a systematic review. EFORT Open Rev. 2019 Jul 17;4(7):476-81.

6. Woodward LJ, Kam PCA. Ankylosing spondylitis: recent developments and anaesthetic implications. Anaesthesia. 2009 May;64(5):540-8.

7. Ak J, Lth J, O P, T H, J A, Le K. Surgical interventions for nephrolithiasis in ankylosing spondylitis and the general population [Internet]. Scandinavian journal of urology. 2015 [cited 2020 Sep 16]. Available from: https://pubmed.ncbi.nlm.nih.gov/26389795/

8. Savill DL. The manubrio-sternal joint in ankylosing spondylitis. J Bone Joint Surg Br. 1951 Feb;33$\mathrm{B}(1): 56-64$.

9. Hollan I, Saatvedt K, Almdahl SM, Mikkelsen K, Moer R, Halvorsen P, et al. Spondyloarthritis: a strong predictor of early coronary artery bypass grafting. Scand J Rheumatol. 2008 Feb;37(1):18-22.

10. Lai C-H, Lai W-W, Chiou M-J, Tsai L-M, Wen J-S, Li C-Y. Outcomes of coronary artery bypass grafting in patients with inflammatory rheumatic diseases: an 11-year nationwide cohort study. J Thorac Cardiovasc Surg. 2015 Mar;149(3):859-866.e1-2.

11. Kobak S, Yilmaz H, Karaarslan A, Yalcin M. Leukocytoclastic vasculitis in a patient with ankylosing spondylitis. Case Rep Rheumatol . 2014;2014:653837. doi:10.1155/2014/653837

12. Patel, S., Sharma, S., Devgarha, S. et al. Complete coronary arterialization in patient of rheumatoid arthritis with fixed deformity of bilateral hips, knees, and kyphosis. Indian J Thorac Cardiovasc Surg 30, 309-310 (2014). 